\title{
El esclavismo antiguo en los Estados Unidos del periodo antebellum (1780-1860)
}

\author{
Clelia MARTÍNEZ MAZA \\ Universidad de Málaga \\ martinezm@uma.es
}

Recibido: 5 de octubre de 2015

Aceptado: 5 de febrero de 2016

\section{RESUMEN}

En los inicios de Estados Unidos como nación, su estructura económica tenía en la mano de obra esclava y su comercio una de sus bases económicas esenciales. Y si la recuperación del mundo antiguo fue esencial en el diseño político de la nueva república, los defensores del esclavismo también acudieron al pasado clásico en busca de un aval ideológico. La esclavitud en Grecia y Roma fue por ello recuperada como antecedente para justificar la práctica. Se pretendía demostrar, mediante la llamada a los clásicos, la necesidad del trabajo esclavo, la continuidad del sistema y los benéficos efectos ya comprobados en el mundo grecorromano, no sólo en el terreno económico, sino también en el ámbito político e incluso artístico.

Palabras clave: Esclavitud. Aristóteles. República agraria. Estados sureños. Modelos clásicos.

\section{Ancient Slavery in the Antebellum United States (1780-1860)}

\begin{abstract}
In the early years of America as a nation, its economic structure had one of its essential bases in slave labour and trade. Besides, if the recovery of the Ancient world was essential in the political design of the new republic, slavery advocates also went to the classical past seeking ideological support. Slavery in Greece and Rome was thus recovered as a precedent to justify the practice. It was intended to demonstrate, by recalling the classics, the need for slave labour, the continuity of the system, and the advantages already proven in the Greco-Roman world, not only in the economic situation, but also in the political and even the artistic field.
\end{abstract}

Keywords: Slavery. Aristotle. Agrarian Republicanism. Southern States. Classical Models. 
A pesar de que la Constitución de los Estados Unidos proclama la igualdad de todos los hombres y la libertad como condición natural del género humano, no incluye una condena explícita a la esclavitud, un término que ni siquiera aparece mencionado. ${ }^{1}$

Es cierto que tampoco encontramos ninguna medida que la ampare, pero no puede negarse que algunos de los preceptos constitucionales reflejan la plena aceptación del esclavismo. En primer lugar, la sección II del art. IV, sobre los fugitivos, donde se declara la obligación de entregar a todo aquel que ha huido y está obligado a trabajar en un estado: "Ninguna persona forzada a prestar servicio o a trabajar en un estado bajo las leyes del mismo, que huya a otro estado, será dispensada de prestar dicho servicio o trabajo amparándose en leyes o reglamentos del estado al cual huyó, sino que será entregada a petición de la parte que tenga derecho a su servicio o trabajo". ${ }^{2}$

En segundo lugar, la protección concedida oficialmente al comercio de esclavos, que se mantuvo por ley hasta 1808 (art. I, sección 9: “el Congreso no podrá prohibir antes del año 1808 la inmigración o importación de aquellas personas cuya admisión sea considerada conveniente por cualquiera de los estados hoy existentes; empero, tal importación podrá ser gravada con un impuesto o derecho que no excederá los diez dólares por cada persona"). Se trató de una condición innegociable que impusieron los delegados de Carolina del Norte en la convención de Filadelfia para aprobar la Constitución. ${ }^{3}$

Por último, el célebre compromiso de los $3 / 5$ exigido por los estados del Sur y que reconocía a los esclavos como parte de la población computable para calcular el número de representantes en las elecciones al congreso. ${ }^{4}$

El reconocimiento tácito del esclavismo en la Carta Magna no resulta extraño pues el nuevo Estado tenía en la mano de obra esclava y su comercio una de sus bases económicas esenciales. Por ello, en la convención reunida en Filadelfia en 1787 para redactar la Constitución, los defensores de este modelo económico consideraron cualquier crítica al trabajo esclavo como una deslealtad a las aspiraciones de los padres fundadores, un ataque al experimento americano destinado a reducir su capacidad económica.

En este ambiente político, en el que el pasado clásico aparece como constante referente ideológico que avala la validez del nuevo ordenamiento constitucional, los esclavistas también presentaron, como justificación de la práctica, los precedentes históricos y, de manera especial, la herencia transmitida desde la Antigüedad clásica, impulsando, como veremos en las páginas siguientes, una hermenéutica propia

1 Este trabajo es la versión escrita y ampliada de la comunicación presentada en el XXXVII coloquio del GIREA que, con el título Hermenéutica de la esclavitud, se celebró en diciembre de 2014 en Ciudad de Méjico. Agradezco a los Dres. Domingo Plácido y Antonio Gonzales las sugerencias allí realizadas. Los errores son responsabilidad mía.

2 Finkelmann 1996, 82-104.

3 Sobre el ambiente de la convención constituyente en el que se fraguó la Constitución y las reivindicaciones de los estados para sostener el nuevo ordenamiento federal vid. MARTínez MAZA 2012.

4 U.S. Constitution, Art. I sec. II § 3: "Tanto los representantes como los impuestos directos serán prorrateados entre los diversos estados que estén integrados en esta Unión, de acuerdo a su respectivo número, el cual se determinará sumando el número total de personas libres; se incluye a los que estén obligados a prestar servicio por determinado número de años y se excluye a los indígenas que no estén sujetos al pago de impuestos; las tres quintas partes de todas las demás personas". 
de la esclavitud en Grecia y Roma. ${ }^{5}$ Para entender esa particular recuperación del esclavismo grecorromano hay que recordar que el acercamiento a este fenómeno histórico fue promovido como estrategia complementaria, como refuerzo de la tesis esclavista que tenía en la tradición bíblica y los argumentos de naturaleza biológica sus recursos más habituales en los estados sureños. ${ }^{6}$ La selección de autores y circunstancias históricas fue, además, previsiblemente arbitraria, como también lo fue la recuperación del escenario político clásico. En efecto, no toda la literatura grecorromana al respecto recibió la misma atención e idéntico interés, ni la recuperación de las reflexiones ofrecidas por el mundo antiguo se realizó de manera sistemática. De las obras manejadas, se seleccionaban, extraían y confeccionaban modelos atemporales desprovistos de todo rigor científico; y así, armados con tales abstracciones políticas, los sureños abordaron el debate abolicionista con instrumentos ajustados a sus intereses. No obstante, esta aproximación no puede ser objeto de condena por su inexactitud, puesto que los intelectuales sureños no pretendieron un estudio profundo de la sociedad grecorromana, ni un análisis teórico, y su acercamiento al esclavismo antiguo tuvo siempre una finalidad eminentemente práctica, manejando a voluntad las fuentes clásicas, en función de sus intereses.

La identificación con el pasado clásico puede comprobarse, por ejemplo, en las palabras de Thomas Roderick Dew (1802-1846), presidente de uno de los más afamados centros educativos del momento - el College de William \& Mary (Virginia) - al afirmar:

se ha defendido que el esclavismo no es favorable a un espíritu republicano pero la historia del mundo entero prueba que está muy lejos de ser así. En las antiguas repúblicas de Grecia y Roma donde el espíritu de libertad se respiraba con mayor intensidad, los esclavos eran más numerosos que los hombres libres... en los tiempos modernos las comunidades que disponen de esclavos desean igualmente la libertad. ${ }^{7}$

Algunos sureños, como el virginiano G. Fitzhugh -autor, entre otras, de la popular Cannibals All! Or slaves without masters, publicada en fechas próximas a la guerra de secesión (1857)-, establecieron incluso una estrecha pero forzada conexión entre sus propios estados y Grecia y Roma al distinguir entre las civilizaciones del Norte y aquellas del Sur, de las que formaban parte tanto ellos mismos como las mediterráneas. ${ }^{8}$

En 1850, James D. B Debow, creador de la prestigiosa revista DeBow, intentaba convencer a las familias sureñas de que en lugar de enviar a sus hijos a los colleges del Norte, en los que, según sus palabras, se promulgaba el abolicionismo, escogieran los centros educativos sureños en los que se mantenía con fuerza la enseñanza clásica. ${ }^{9}$ Sostenía además ese paralelismo entre mundo clásico y estados del Sur al afirmar

5 Malamud 2009, 76; Richard 2009, 181-204.

6 Por ejemplo, los propuestos en Debow 1850a, 281-286; CARTwright 1851, 28-35.

7 Dew 1853, 461, publicado en un volumen de ensayos junto a las contribuciones de otros reputados esclavistas. Desarrolla una reflexión muy similar en Dew 1832. Una postura próxima mantiene Rivers 1893.

8 Fitzhugh 1860, 369-381.

9 Debow 1856, 2; Miles 1971, 263-265; Durrill 1999, 496-497. 
que "la civilización en el mundo tiene su origen en el Sur, como queda reflejado en la Historia. Grecia, Roma y los estados esclavos del Sur han dado al mundo entero la civilización, las artes, literatura, leyes y el gobierno". ${ }^{10}$

Entre los esclavistas más combativos, Fitzhugh recuperó, en defensa del trabajo esclavo, su existencia en el mundo grecorromano y lo interpretó como un instrumento beneficioso no sólo en el ámbito económico, sino también en el sociopolítico e ideológico. Así, en la mentalidad americana la esclavitud abandonaba su naturaleza de mal menor, tal y como la justificaban muchos esclavistas, para modelarse a la manera del mundo antiguo, o expresado con propiedad, a la manera en que a los esclavistas les interesaba recuperar el modelo clásico.

Fitzhugh llegó al absurdo de intentar trazar las huellas que mostraban la vinculación existente, por un lado, entre los colonos que se habían asentado en lo que entonces era el Sur de EE.UU. y los antiguos romanos, y, por otro, entre sus adversarios yankees y los siervos anglos-sajones. ${ }^{11}$ Incluso los sureños observaban con cierta preocupación el hecho de que Grecia y Roma hubieran sido conquistadas por enemigos del Norte, además, con un menor grado de civilización. Así lo recoge W. J. Rivers en el Daily South Carolinian en 1855:

Si hay que destacar un momento de la historia de Grecia por encima de los demás es aquel en el que observamos la gradual historia de la destrucción de su libertad por el enemigo del Norte, un trabajo comenzado por Filipo de Macedonia y completado por Alejandro... Nosotros, también sufrimos la peligrosa amenaza del Norte; nosotros también tenemos un nombre y una herencia de libertad que defender. ${ }^{12}$

Pero no sólo acudía al ejemplo clásico para defender la necesidad del trabajo esclavo y sus benéficos efectos en Grecia y Roma, sino también para demostrar la continuidad del sistema. Entendida como una institución continuada en el tiempo, se aducía entonces que, como todo lo perdurable, debía ser natural y al ser natural, en consecuencia, el esclavismo era bueno. Por otro lado, la deducción era idéntica cuando se recordaba el carácter universal de la esclavitud, ya que cualquier cosa universal debía ser natural y, por lo mismo, de nuevo buena. ${ }^{13}$ Incluso algunos esclavistas, como el sureño que se hacía llamar "Southron", daban un paso más y añadían que como en el admirado mundo grecorromano existían esclavos, la esclavitud debía ser, por lo tanto, buena. ${ }^{14}$ La esclavitud aparece entonces como parte del orden natural y las propuestas abolicionistas se tildan de creencias erráticas que provocarían discordia social, y se perciben como más peligrosas si cabe en la medida en que estaban alterando la naturaleza misma de las cosas.

En efecto, los esclavistas consideraban que la ausencia de un movimiento abolicionista en el pasado en general y en el mundo antiguo en particular, constituía indicio suficiente de que el activismo antiesclavista que surgió a finales del XVIII y comien-

10 Deвow 1850, 15-19.

11 Fitzhugh 1854, 241-243.

12 Rivers 1855.

13 Así lo defendía Debow: Skipper 1958; Hall 1982, 97-104; CobB 1858, 3-5; Finkelman 1999, $75-115$.

14 Southron 1838, 737. 
zos del XIX debía ser considerado una anomalía, pues no existían antecedentes que legitimaran la abolición. La Historia demostraba que la esclavitud formaba parte del orden natural en el que se articulaba la sociedad y allí donde se constataba la decadencia del sistema (innegable por otro lado) se justificaba no como resultado de una agitación popular basada en la moralidad del abolicionismo, sino como respuesta a las necesidades económicas, pues el abandono del esclavismo debía estar siempre fundado en principios de mercado.

En la defensa del esclavismo como parte del orden moral en el que se basaba la sociedad, los intelectuales del Sur encontraron una poderosa arma en Aristóteles. ${ }^{15}$ En especial se recuperó su Política (I.2), que no casualmente fue objeto de una nueva edición en griego y latín que se sumó a las tres versiones en inglés disponibles durante este período (1830-1850). ${ }^{16}$ Fue constantemente enarbolada la máxima aristotélica sobre la esclavitud, al mismo tiempo natural y beneficiosa, conectada con la regla universal que establecía relaciones de poder de los humanos sobre los animales, los adultos sobre los niños y los hombres sobre las mujeres. El señor se distinguía de su esclavo no sólo por su mayor inteligencia (1.5), sino también por su gran amor por la libertad, que es un requisito para un ciudadano de una república: "porque es por naturaleza un esclavo quien es capaz de pertenecer a otro y hace por pertenecer a otro". Estas y otras reflexiones convirtieron a Aristóteles en el filósofo más admirado por los intelectuales del Sur. Así "Southron" escribía en 1838:

Apelamos con plena confianza a Aristóteles, uno de los más profundos filósofos de la Antigüedad... Aristóteles ha declarado expresamente que en su natural estado, desde el origen de las cosas, una parte de la familia humana debería ordenar y el resto obedecer, ${ }^{17}$ y que esta distinción entre dueño y servidor es una distinción natural e indispensable, y cuando encontramos que existe entre libres y esclavos, no es el hombre sino la naturaleza la que ha ordenado esta distinción. ${ }^{18}$

En 1840, J. C. Calhoun -líder sureño y vicepresidente de EE.UU. en 1832- recomendaba el estudio de la Historia Antigua y la lectura de los principales tratados sobre el gobierno, entre los cuales Aristóteles ocupaba un lugar preferente. ${ }^{19}$ Elogios similares recibió el filósofo griego de G. Frederick Holmes (1820-1897), profesor de clásicas en varias universidades sureñas como los centros de Richmond, Missisipi y Virginia: "cuanto más estudio Aristóteles menos necesito descubrir otro filósofo". ${ }^{20}$ Fitzhugh también lo consideraba el filósofo más sabio de todos los tiempos: "La teoría de Aristóteles, promulgada hace más de 2000 años, generalmente considerada cierta durante 2000 años, y destinada, así lo esperamos, a que sea aceptada pronto

15 Wiltshire 1987, 9-10; Monoson 2011, 248-249.

16 Monoson 2011, 255-256.

17 Idéntica interpretación del texto aristótelico fue recogida por HoLmes 1850, 194.

18 Southron 1838, 737-747. El autor rechaza explícitamente las teorías propuestas por los pensadores ilustrados franceses entre los que cita a Montesquieu y Rousseau.

19 Calhoun 1983.

20 Holmes 1855, 618-622. 
como la única teoría de gobierno y social válida" ${ }^{21}$ En 1855, admitía con cierta sorpresa a G. Frederick Holmes que había plagiado a Aristóteles sin leerlo: "me he dado cuenta de que no sólo he adoptado sus teorías, sus argumentos, y sus ejemplos, sino incluso sus palabras". ${ }^{22}$

Pero el concepto aristotélico de esclavitud natural fue ajustado a la realidad inmediata de los patronos sureños y, para obtener un modelo ideológico que legitimara su práctica, los esclavistas incorporaron el componente racial. ${ }^{23} \mathrm{~W}$. J. Grayson (17881863), miembro de la Cámara de Representantes por Carolina del Sur, escribió en la revista DeBow's:

La máxima de Calhoun es que no puede existir un gobierno democrático a menos que la clase trabajadora esté constituida por esclavos... y no es cosa nueva, sino que ya fue así hace 2000 años. ${ }^{24}$ Tampoco Mr. Calhoun es el primero que lo menciona, sino Aristóteles en su Política, un texto que debería ser leído en todos los colegios del Sur y en el que establece la máxima de que un hogar o una comunidad completa se compone de hombres libres y esclavos. Aristóteles escribe para las democracias y mantiene que los esclavos deberían ser bárbaros y no griegos. Y Mr. Calhoun también apunta ahora como ventaja el hecho de que los esclavos del sur sean de raza negra, una raza bárbara suficientemente fuerte y dócil para el trabajo. Pero la proposición completa en lo relativo a la esclavitud como a la raza de los esclavos ya fue establecida por el filósofo griego..$^{25}$

En efecto, Calhoun, que tras dimitir como vicepresidente fue líder de la minoría esclavista en el senado entre 1830-1840, se erigió en uno de los más fervientes defensores de la esclavitud entendida no como mal necesario, sino como un activo beneficioso para el país. En sus propuestas podemos comprobar esos ecos aristotélicos que reconocen sus correligionarios:

Es un enorme y peligroso error suponer que todo el mundo es igualmente adecuado para la libertad. Se trata de una recompensa que debe ser ganada, no una bendición obtenida gratuitamente, una recompensa reservada al inteligente, al patriota, al virtuoso y al merecedor de ella y no una bendición otorgada a gente demasiado ignorante, degradada, viciosa, para ser capaz de apreciarla y disfrutarla. ${ }^{26}$

Para Fitzhugh los afroamericanos respondían al estereotipo de esclavitud natural aristotélico incluso de manera más ajustada que los esclavos griegos y romanos:

21 Una reflexión similar expresa W. Harper, presidente del tribunal supremo de Carolina del Sur (ver HARPER 1836, 16); GraYSON 1860, 48-66.

22 Fitzhugh 1857, 9; ID. 1855.

23 Calhoun 1953, 42-44; Wish 1949, 259-260; Wiltshire 1977, 36. En este desarrollo del componente racial tuvieron gran influencia los trabajos de J. Clark Noтт 1854.

24 El argumento de la antigüedad del sistema esclavista fue desarrollado asimismo por Holmes 1850, 194.

25 GraYson 1860, 48-49; también Совв 1858, xl-1xvii y 15-17.

26 Calhoun 1837. 
Hay un poderoso argumento a favor de la esclavitud de los negros por encima de cualquier otro: que el negro, incapaz para las artes, el comercio, y cualquier otro propósito que requiera habilidad, acepta que estas actividades estén en manos de los blancos, y no provoca el descrédito de la industria, como en Grecia y Roma, donde los esclavos no fueron sólo artistas y técnicos, sino también comerciantes. ${ }^{27}$

La subordinación de los afroamericanos se justificaba por su condición inferior, que Fitzhugh comparaba a la de un niño. ${ }^{28} \mathrm{Y}$ así reivindicaba: "la democracia ateniense no es posible en una nación negra, ni el gobierno de la ley es el adecuado para el individuo negro. Él no es sino como un niño y debe ser gobernado como un niño". En otras ocasiones, Fitzhugh los asimilaba a los salvajes, otra categoría de gente incapaz del autogobierno: "los griegos y romanos eran muy pródigos empleando el término bárbaro, pero dudamos de si vieron alguna vez a un salvaje. Heródoto habla de un pueblo sin cabezas y con los ojos en el pecho, pero no dice ni una palabra de hombres de piel negra y sin ley". ${ }^{29}$

Dado que los afroamericanos eran criaturas infantiles que no estaban preparadas para actuar con iniciativa propia, la posesión de esclavos se justificaba como forma de control social necesaria. Los blancos del Sur se reconocían como individuos benévolos que cumplían un papel fundamental, al cuidar de sus esclavos y disciplinarlos como un padre hace con un niño que no puede asumir ninguna responsabilidad y actuar de manera independiente sin supervisión adulta. El amo era también mentor y maestro e, incluso, algunos sureños evocan la plantación o la granja como una escuela donde debían ejercer su tutela ${ }^{30}$ y los esclavos sureños eran más sanos y más felices que cualquier otro trabajador o campesino que hubiera habido sobre la faz de la tierra. ${ }^{31}$

Por otro lado, la recuperación del mundo clásico sirvió para avalar argumentos en defensa del esclavismo de naturaleza muy dispar. En primer lugar, se atiende a los criterios más evidentes, los de orden económico. Los esclavistas consideraban una deslealtad hacia los estados del Sur cualquier crítica diseñada para reducir la capacidad económica de la nueva nación. Resultaba intolerable que una reacción de naturaleza emocional, como la que subyacía en el movimiento abolicionista, pudiera influir e incluso determinar la buena marcha de la economía del país. El valor de los esclavos como fuerza de trabajo necesaria para mantener un cierto nivel de producción quedaba fuera de toda duda y remontaron este principio al mundo clásico. Fitzhugh refrendaba esta percepción acudiendo a la historia de los estados más poderosos y duraderos de la Antigüedad, sociedades que habían usado el trabajo esclavo a gran escala:

27 Fitzhugh 1857, 297.

28 Siguiendo a Aristóteles Pol. I 1260a; Fitzhugh 1854, 83; Upshur 1839, 685-686. Argumentos fisiognómicos en HanNAFORD 1996, 57; MaIlloux 2002, 114.

29 Fitzhugh 1854, 232.

30 Fitzhugh 1854, 230-231; ID. 1857, cap. 1.

31 Southern Agriculturist 1832, 562; ibid. 1833, 643; Southern Cultivator 1845, 148. 
Es de sobra conocido que la gran y perpetua arquitectura de la Antigüedad fue resultado de la esclavitud. El esforzado trabajo colectivo que requería la construcción, cuando las técnicas estaban tan poco avanzadas, sólo pudo llevarse a cabo gracias a la intervención de una autoridad despótica, como la de un amo hacia sus esclavos. ${ }^{32}$

Fitzhugh consideraba que el modelo desarrollado por los estados abolicionistas como alternativa al esclavismo, el capitalismo industrial que explotaba a los obreros, era una especie de "canibalismo moral". ${ }^{33}$ No proporcionaba un sistema de seguridad económica a los trabajadores del Norte similar al que ofrecía el paternalismo de la esclavitud sureña. Defendía que la industrialización de Gran Bretaña y de los estados abolicionistas constituía una forma nueva y más dura de esclavitud:

La libertad en Inglaterra, como en Roma y en Grecia, es como lo fue entonces, el privilegio de unos pocos, no el derecho de muchos. Pero si en Grecia, Roma y los estados del sur de América, la gente ha ganado en protección lo que ha perdido en libertad, en Inglaterra en cambio, no tiene ni libertad ni protección. Es verdad que los trabajadores de las fábricas, a diferencia de los esclavos, podrían moverse de un lugar a otro, pero lo que harían en realidad sería moverse de una situación desesperada a otra. ${ }^{34}$

$\mathrm{Su}$ análisis obviaba conscientemente que las plantaciones del Sur, lejos de ser enclaves que evocaban la vida pastoril, idílicos frente a la pésima imagen del mercantilismo, eran empresas con propósitos igualmente comerciales, con cultivos destinados a la producción a gran escala que satisfacían la demanda de esas mismas fábricas textiles objetivo de su crítica. ${ }^{35}$

Conforme las voces a favor del abolicionismo se hicieron más fuertes, mayor fue el número de argumentos incorporados a la réplica esclavista en los que la experiencia clásica legitimaba su postura. Además de los beneficios económicos de la esclavitud, tal y como quedaba comprobado en los grandes estados de la Antigüedad clásica, se atribuyen efectos igualmente positivos en el ámbito político, pues el esclavismo garantizaba la igualdad y la libertad del cuerpo ciudadano. ${ }^{36}$ Los intelectuales sureños se erigieron así en guardianes del modelo republicano clásico. ${ }^{37} \mathrm{En}$ el desarrollo de esta línea argumental, no recuperaron exclusivamente los modelos políticos del mundo antiguo más admirados en la América del período constituyente, la república romana y Esparta -considerados como el mejor espejo al que podían acudir para articular el ordenamiento interno de su gobierno- sino que acudieron en reiteradas

32 Fitzhugh 1854, 89.

33 Fitzhugh 1857, cap. 1. Años antes lo había formulado de la siguiente manera: "liberty and equality are new things under the sun. The free states of Antiquity abounded with slaves" (FITZHUGH 1854, 226).

34 Fitzhugh 1857, 117.

35 Hartz 1955, 145-200; Hofstadter 1973, 86-117; Appleby 1982, 833-849; Reidy 1992, 58-80.

36 MCCord 1851, 357; A. Jackson: "There never was a Government on the face of the earth but what permitted Slavery. The purest sons of Freedom in Grecian Republics, the citizens of Athens and Lacedaemon, all held slavery" (Anales del Congreso; primera sesión, 12 de febrero de 1790, véase GALES (ed.), 1834, 1242); HAMmOND 1858, 962: "in all social systems there must be a class to do the menial duties, to perform the drudgery of life".

37 Calhoun 1837; Tyse 1987, 347-362; Montgomery 1994, 126; Fink 1999, 122-123. 
ocasiones a Atenas. Sorprende en cierta medida la nueva incorporación, pues esta polis fue desdeñada inicialmente como modelo político a causa de la inestabilidad y la violencia que padecía, resultado de los excesos de una democracia directa. ${ }^{38}$

El cambio en la percepción del modelo ateniense guarda relación con las nuevas formas más democráticas que empiezan a promoverse desde el gobierno federal, una vez establecido y consolidado el nuevo Estado. ${ }^{39}$ A partir de 1790, la clase política liderada por Jefferson y Madison empezó a introducir en el gobierno -y a aprobarmedidas más democráticas, como la supresión del criterio timocrático en el derecho a voto, ${ }^{40} \mathrm{o}$ la eliminación de la propiedad como requisito imprescindible en las votaciones; ${ }^{41}$ a la vez se acentuó el papel crucial de la representación, si bien nunca se pretendió, a través de estas formas más democráticas, evolucionar hacia una democracia directa al estilo de la ateniense.

Una vez rehabilitada, Atenas se erigió en el paradigma clásico de los estados del Sur, junto a Roma, que mantuvo su entidad como referente político. ${ }^{42}$ No obstante, los intelectuales sureños no desarrollaron una reflexión individualizada y precisa que permita despejar los motivos por los que, en unos casos, el modelo seleccionado fue el romano y, en otros, el ateniense. Incluso, con frecuencia, ambos fueron recuperados de manera conjunta. En la novedosa identificación con Atenas se aducía la igualdad concedida a todos los adultos varones ciudadanos que, al igual que ellos, disfrutaban en común de los beneficios derivados del establecimiento del esclavismo. ${ }^{43}$ El ejemplo ateniense servía ahora para ennoblecer su experiencia y dignificar sus acciones. La libertad e igualdad política se presentaban como ejemplo de las bondades derivadas de la esclavitud. ${ }^{44}$ Fitzhugh escribió:

la esclavitud encumbra a los blancos que son pobres. Porque lejos de ser la base de la sociedad como en el Norte, donde son jornaleros, obreros no especializados o buscan en la basura, los convierte en ciudadanos privilegiados, como los ciudadanos griegos y romanos que tienen bajo ellos una nutrida clase. ${ }^{45}$ En una sociedad esclava, un blanco no es superior a otro porque todos son iguales en privilegios. Pensamos que la clase gobernante debería ser suficiente numerosa para atender a todos los intereses, y situados en esa posición de privilegio, representarlos de manera adecuada... Griegos y romanos se encontraban en esa misma posición, como los barones de Inglaterra y ahora los blancos del Sur. Si no son todos de la misma condición como en Grecia y Roma, al menos pertenecen a la misma raza, la de los dueños y tienen derechos de ciudadanía

38 Martínez Maza 2010, 215-226; EAD. 2012, 145-177; al mismo tiempo, triunfa, por ejemplo, en la estética arquitectónica, el estilo neoclásico: HAMLIN 1944, 266; RoBERTs 1994.

39 Matthews 1984, 77; JefFERSON 1816.

40 Mantenido hasta entonces y defendido desde los primeros tiempos de la Revolución: Records of the Federal Convention, June 29, 1787.

41 La propuesta fue defendida por Gouverneur Morris el 7 de Agosto de 1787: FARRAND 1911, vol. II, 299.

42 HARPER 1836, 14-15. Esparta fue pronto descartada como paradigma político con posibilidades de aplicación práctica tal y como argumentan: ADAMs 1851 [original de 1787], 549-556 (Lacedaemon); HAMILTON 1787; ID. 1904 [original de 1781]; AMEs 1809; JEFFERSON 1823.

43 Rivers 1858, 13-19; DurRill 1999, 498.

44 DEBOW 1860, 174-175.

45 Fitzhugh 1857, 320; Una crítica similar realiza Hammond 1858, 962. 
y privilegios exclusivos, y ningún interés en que este derecho de ciudadanía se viera extendido, perturbado y convertido en algo despreciable o sin valor. ${ }^{46}$

\section{Y añadió:}

no necesitamos esclavos blancos en el Sur porque ya tenemos a los negros. Nuestros ciudadanos como los de Grecia y Roma son una clase privilegiada. Deberíamos adiestrarlos y educarlos para ganarse estos privilegios y que ejercieran los deberes que la sociedad les confiere ${ }^{47} \ldots$ Es una distinción ser un sureño, como una vez lo fue ser ciudadano romano... los abolicionistas dicen que una de las irremediables consecuencias de la esclavitud es que los pobres son abandonados. No era así en Atenas ni en Roma ni debería ser así tampoco en el Sur... Debemos proporcionar una ocupación honorable a todos nuestros ciudadanos mientras cultivamos y mejoramos sus mentes para pedirles que tomen parte en la administración de justicia y gobierno. Deberíamos hacer la pobreza tan honorable como lo fue en Grecia y Roma. Porque ser un virginiano debería ser una distinción más alta que la riqueza o cualquier título otro otorgado. ${ }^{48}$

Junto a este sentimiento de solidaridad de clase, igualdad y camaradería entre el cuerpo ciudadano, para el que los esclavistas buscaron un referente de prestigio incontestable en el mundo clásico, un segundo beneficio en el ámbito político que los sureños reconocían en el esclavismo era la estabilidad y la perdurabilidad que había proporcionado a las antiguas repúblicas, cualidades que se consideraban esenciales para un gobierno tan joven. ${ }^{49}$ W. J. Rivers, profesor de literatura griega en el College de Carolina del Sur afirmaba: "en ningún lugar podríamos encontrar a gente más parecida a los griegos que en nuestro propio pueblo". Reivindicaba que si los Estados Unidos hacían caso omiso a los abolicionistas además "de proteger el esclavismo y sus efectos como la consolidación del espíritu patriótico, la nación tendría más paz y prosperidad. En caso contrario, la inestabilidad provocada en la república conduciría a la tiranía". 50

Los intelectuales sureños, sin embargo, sí acudieron al ejemplo de Roma para defender, en este caso, que la abolición de la esclavitud y sus terribles consecuencias (inestabilidad, tiranía, ruina económica) podían evitarse, paradójicamente, reforzando la clase de pequeños propietarios, porque fue la disolución del pequeño campesinado la que favoreció, en primer lugar, la opresión patricia, y, en segundo lugar, la consecuente rebelión de los ciudadanos desfavorecidos, "hombres libres convertidos en instrumentos cómplices de demagogos sin principios" $" 51$ que terminarían por derrocar el esclavismo y el gobierno de la república. ${ }^{52}$ Por esta razón, la mayor parte de los sureños aplaudieron las reformas de los Graco, a pesar de considerar discutibles sus métodos, pues su objetivo era recuperar el tejido social agrícola mediante la redis-

46 Fitzhugh 1857, 357.

47 Fitzhugh 1854, 93.

48 Fitzhugh 1854, 255-256.

49 Calhoun 1837, 12-16; Tyse 1987, 347-362; Gourevitch 2014, 18-40.

50 Dew 1832; sobre los beneficios políticos del esclavismo: CALHOUN 1837, 14.

51 Swinton Legaré 1846, 518-519.

52 Fisk 1835, 9; PeSSEN 1967, 92-94; Roediger 2007, 74-77. 
tribución de tierras. ${ }^{53}$ Sólo mediante una amplia base de pequeños propietarios podía garantizarse el éxito de la república esclavista. Y en un momento en el que resulta factible incrementar el territorio de la nación con relativa facilidad, se podía asegurar la coexistencia de una amplia base de pequeños propietarios y el mantenimiento del trabajo esclavo.

Como argumento adicional se exhibían los logros intelectuales y artísticos de las repúblicas clásicas, y muy especialmente los de Atenas, resultado de los efectos benéficos de la esclavitud:

Estamos en deuda con Grecia, Roma, Egipto, y los restantes estados de la antigüedad por su gran prosperidad y civilización, prosperidad y civilización que parece más milagrosa aún cuando observamos su ignorancia en ciencias físicas. Este alto grado de civilización y la esclavitud doméstica no coexisten, sino que son efecto y causa. El intelectual con inclinación por la Historia Antigua y la literatura comprueba que Grecia y Roma son deudoras de esta institución (el esclavismo) solo por el gusto, el placer y el provecho de cultivar sus mentes y sus corazones. Si tales intelectuales hubieran estado ligados a las nociones yankees del ahorro, habrían producido un Franklin, con su "penique ahorrado, penique ganado", habrían tenido filósofos utilitaristas, inventado las máquinas de hilar, pero nunca habría surgido un poeta, un orador, un escultor o un arquitecto, nunca habrían creado una obra de arte única. Las lejanas y solitarias ruinas del arte griego y romano, la columna dórica y las agujas del gótico, el genio y la energía de la sociedad en la que surgió el esclavismo, son el noble resultado de la esclavitud doméstica. ${ }^{54}$

Más adelante, Fitzhugh identifica a los más destacados intelectuales americanos con grandes héroes grecorromanos, ambos fruto de la esclavitud: "Escipión y Arístides, Calhoun y Washington son la noble consecuencia de la esclavitud doméstica". ${ }^{55}$ Fitzhugh suponía que el elevado grado de civilización de los atenienses hundía sus raíces en la esclavitud:

Deberíamos, como los atenienses, ser el pueblo más cultivado del mundo, y la mejor manera de conseguir ese resultado es empleando a nuestros blancos en la ingeniería (y otros ámbitos tecnológicos relacionados con la mecánica), ${ }^{56}$ el comercio y otras actividades profesionales y confinar a los negros a labores de granja y a trabajos mecánicos. ${ }^{57}$

Las reflexiones de Fitzhugh sobre los benéficos efectos de la esclavitud eran compartidas por todos los intelectuales sureños. Thomas Dew señalaba que "las leyes de Licurgo se promulgaron, se escuchó la sublime elocuencia de Demóstenes y Cicerón y se asistió a las gloriosas hazañas de Epaminondas y Escipión en naciones en las que existía el esclavismo y en las que en ningún momento desaparecieron los vínculos

53 Holmes 1855, 617-618; Genovese 2002, 466.

54 FitZHUGH 1854, 242.

55 Fitzhugh 1854, 244.

56 Disciplinas que en el s. XIX eran comparables a las artes.

57 Fitzhugh 1854, 241-244; defiende la inferioridad intelectual de los negros Coвв 1858, 32-38. 
que unían a amos y esclavos". ${ }^{58}$ Del mismo modo, G. Frederick Holmes recordaba que el período de esplendor espartano se sostenía en el esclavismo, y que, durante el período de gloria y de grandes triunfos atenienses, la agricultura, el comercio, las finanzas y las manufacturas estuvieron en manos esclavas; que mientras Esquilo componía sus tragedias y Píndaro sus odas, Tucídides su Historia y Platón exponía sus reflexiones filosóficas, mientras vivían Demóstenes y Aristóteles, el esclavismo era universal. Los poseedores de esclavos romanos habían conquistado el mundo, legislado en momentos sucesivos de la Historia y sobre ellos se erguía la civilización y las instituciones modernas. ${ }^{59}$

En definitiva, los esclavistas realizaron una lectura muy particular del pasado grecorromano y sustentaron en el trabajo esclavo incluso su progreso en el ámbito artístico, político y ciudadano. Desde su perspectiva, cualquier desafío a la legitimidad del esclavismo debería abordar, en primer lugar, una denuncia de todos los antecedentes intelectuales presentes ya en el mundo clásico que justificaban y racionalizaban la práctica y para rebatir el peso de la tradición grecorromana, el movimiento abolicionista no disponía de más recursos ideológicos que el sentimentalismo.

Tras la guerra de secesión, en la nueva era industrial, la economía, la ingeniería, las ciencias, las llamadas artes útiles, y el alemán y el francés, frente al latín y el griego, serán los referentes de la nación. El tiempo de una sociedad agrícola llegó a su fin y con él, el empleo de los clásicos en América. ${ }^{60}$ En un mundo plenamente industrializado poco amparo ideológico podían ofrecer. El 6 de diciembre con la decimotercera enmienda, aprobada en 1865 , la esclavitud era prohibida por mandato constitucional. ${ }^{61}$

58 Dew 1832, 32-33.
59 Holmes $1855,617$.

60 El incuestionable cambio de paradigma ha sido ampliamente estudiado y exhaustivamente analizado tal y como revela la vastísima bibliografía al respecto. Entre otros estudios recientes, cabe mencionar: WINTERER 2002, 101: "As the modern university rose, the classical languages were dethroned. The proliferation of new studies in the curriculum, such as modern languages, modern history, and social sciences, as well as the advent of elective study, helped to push Latin and especially Greek to the side"; ibid. 107: "New conceptions of history also eroded the relevance of antiquity to 'modern' civilization. In the late nineteenth century the first generation of professional social scientists in the universities adopted a new conception of history and America's place within it. This new historical paradigm offered the present rather than the distant past as social guide, a view that not only undermined antiquity's claim to authority but advertised the findings of social science as eminently more useful for current concerns". RICHARD 2009, 207-208: "The Civil War seems to have been crucial in inaugurating the decline of the classics... In 1886 Harvard eliminated its Greek entrance requirement... Other older universities like Yale, as wells as new universities like Cornell, Johns Hopkins, and the University of Chicago, followed suit in the ensuing decade, as universities moved toward curricula that allowed greater specialization business, science, and other "majors"... As the percentage of educated Americans studying the classics decreased, the role of classical knowledge in the political culture declined". Ya en 1984, M. Reinhold, padre de los estudios sobre la recepción de los clásicos en EE.UU., resumía el proceso de esta manera (la cursiva es mía): "Greek was a victim of the sense of progress of the nineteenth century, which demanded not only a place for new subjects in the curriculum but a sharp symbolic break with the past" (REINHOLD 1984, 333).

61 Enmienda 13 sección 1 (1865): "Ni la esclavitud ni la servidumbre involuntaria existirán en los Estados Unidos o en cualquier lugar sujeto a su jurisdicción, salvo como castigo por un delito del cual la persona haya sido debidamente convicta". 


\section{Bibliografía}

Adams, J. (1851): "The Defence of the Constitution of Government of the United States of America, against the Attack of M. Turgot, in His Letter to Dr. Price, Dated on TwentySecond Day of March, 1787", [en] Ch. F. Adams (ed.), The Works of John Adams, Second President of the United States: with a Life of the Author, Notes and Illustrations, vol. IV, Boston, 271-588.

Ames, F. (1809): "Hints and Conjectures Concerning the Institutions of Lycurgus", Works of Fisher Ames, Compiled by a Number of his Friends, Boston.

Appleby, J. (1982): "Commercial Farming and the 'Agrarian Myth' in the Early Republic", Journal of American History 68, 833-849 (http://dx.doi.org/10.2307/1900771).

Calhoun, J. C.

(1837): Speech on the Reception of Abolition Petitions (US Senate).

(1953): A Disquisition on Government and Selections from the Discourse, Indianapolis (ed. C. Gordon Post).

(1983): "Letter to A.D. Wallace (17 december 1840)", [en] C. N. Wilson (ed.), The Papers of John C. Calhoun, vol. XV, Columbia, 389.

CARTwright, S. A. (1851): "Report on the diseases and physical peculiarities of the Negro Race", The New Orleans Medical and Surgical Journal, 691-715.

Coвb, T. R. R. (1858): An Inquiry into the Law of Negro Slavery in the United States of America. To Which is Prefixed, An Historical Sketch of Slavery, Athens (GA)-London.

Debow, J. D. B.

(1850): "The Origin, Progress, and Prospects of Slavery", Debow's Review, Agricultural, commercial, industrial progress and resources 9, 9-19.

(1850a): "Slavery and the Bible", Debow's Review, Agricultural, commercial, industrial progress and resources (unsigned) 9, 281-286.

(1856): "Note", The People's Press from Winston-Salem, North Carolina, 28 November.

(1860): The Interest in Slavery of Southerner Non-Slaveholders, Charleston.

Dew, T. R. (1832): Review of the Debate in the Virginia Legislature of 1831 and 1832, Richmond.

Dew, T. R. ET ALII (1853): "Professor Dew on Slavery", [en] The proslavery Argument. As maintained by the most distinguised writers of the Southern States, Philadelphia, 287-490.

Durrill, W. K. (1999): “The Power of Ancient Words: Classical Teaching and Social Change at South Carolina College, 1804-1860", The Journal of Southern History 65/3, 469-498.

FARRAND, M. (1911): The Records of the Federal Convention of 1787, New Haven, III vols.

FinK, L. (1999): "From Autonomy to Abundance: Changing Beliefs about the Free Labor system in Nineteenth Century America", [en] S. L. Engerman (ed.), Terms of Labor: Slavery, Serfdom and Free Labor, Stanford, 116-136.

Finkelmann, P.

(1996): Slavery and the Founder: Race and Liberty in the Age of Jefferson, New York.

(1999): "Thomas R. Cobb and the Law of Negro Slavery", Roger Williams University Law Review 5, 75-115.

Fisk, T. (1835): Capital against Labor: An Address Delivered at Julien Hall before the Mechanics of Boston on Wednesday Evening, May 20, Boston.

FITZHUGH, G. 
(1854): Sociology for the South, or the Failure of Free Society, Richmond.

(1855): "Letter to George F. Holmes (april 11, 1855)", [en] George Frederick Holmes Papers. Box 1: Correspondence and Papers, 1815-1901 and undated. Letterbook 1835-1864, David M. Rubenstein Rare Book \& Manuscript Library, Duke University, Durham.

(1857): Cannibals All or Slaves without Masters, Richmond.

(1860): "Superiority of Southern Races", Debow's Review, Agricultural, commercial, industrial progress and resources 31/4, 369-381.

Gales SR., J. (ED.), (1834): The Debates and Proceedings in the Congress of the United States, Washington.

Genovese, E. D. (2002): "The Gracchi and Their Mother in the Mind of American Slaveholders", Journal of the Historical Society 2, 455-482.

Grayson, W. J. (1860): “Mackay's Travels in America. The Dual Form of Labor”, Debow's Review, Agricultural, commercial, industrial progress and resources 28/1, 48-66.

Gourevitch, A. (2014): From Slavery to the Cooperative Commonwealth. Labor and Republican Liberty in the Nineteenth Century, Cambridge (http://dx.doi.org/10.1163/24058 36X-00101008).

Hall, M. (1982): “The Proslavery Thought of J.D.B. Debow: A Practical Man's Guide of Economics", Southern Studies 21, 97-104.

Hamilton, A.

(1787): "Concerning Dangers from Dissensions Between the States", The Federalist Papers 6 , November 14 .

(1904): “The Continentalist, July 12, 1781", [en] H. C. Lodge (ed.), The Works of Alexander Hamilton, vol. I, New York-London, 243-289.

Hamlin, T. (1944): Greek Revival Architecture in America, Oxford.

Hammond, J. R. (1858): "Mudsill Speech", The Congressional Globe, 4 March.

HANNAFORD, I. (1996): Race: The History of an Idea in the West, Baltimore.

HARPER, W. (1836): Anniversary oration delivered by the Hon. William Harper in the Representative Hall, Columbia S.C., Dec. 9, 1835, Washington.

Hartz, L. (1955): The Liberal Tradition in America, New York.

Hofstadter, R. (1973): "Calhoun: The Marx of the Master Class", [en] The American Political Tradition and the Men Who Made It, New York, 86-117.

Holmes, G. F.

(1850): "Observations on a Passage in the Politics of Aristotle Relative to Slavery", Southern Literary History 16/4, 193-205.

(1855): “Ancient Slavery", Debow's review, Agricultural, commercial, industrial progress and resources 19/6, 617-637.

JefFerson, T. (1816): "Letter to Isaac Tiffany, August 26", [en] A. E. Bergh - A. A. Lipscomb (eds.), The Writings of Thomas Jefferson, Washington (1907) vol. XV.

(1823): "Letter to Adamantios Coray, October 31", [en] A. E. Bergh - A. A. Lipscomb (eds.), The Writings of Thomas Jefferson, Washington (1907), vol. XV.

MaIlloux, S. (2002): "Re-marking slave bodies, Rhetoric as Production and Reception", Philosophy and Rhetoric 35, 96-119 (http://dx.doi.org/10.1353/par.2002.0007).

Malamud, M. (2009): Ancient Rome and Modern America, Oxford (http://dx.doi. org/10.1002/9781444305074). 
Martínez MazA, C.

(2010): "Democracia ateniense vs. revolución americana: el rechazo al paradigma clásico", Potestas 3, 215-226 ( http://dx.doi.org/10.1163/2405836X-00101008).

(2012): El espejo griego: Atenas, Esparta y las ligas griegas en la América del periodo constituyente (1786-1789), Barcelona.

Matthews, R. K. (1984): The Radical Politics of Thomas Jefferson: A Revisionist View, Lawrence.

McCord, D. (1851): "How the South is Affected by Slavery Institutions", Debow's Review, Agricultural, commercial, industrial progress and resources 11, 349-363.

Miles, E. A. (1971): "The Old South and the Classical World", North Carolina Historical Review 48, 258-275.

Monoson, S. S. (2011): "Recollecting Aristotle. Pro-Slavery thought in Antebellum America and the Argument of Politics Book 1", [en] E. Hall et alii, Ancient Slavery and Abolition: from Hobbes to Hollywood, Oxford, 247-277 (http://dx.doi.org/10.1093/acprot:o so/9780199574674.003.0009).

Montgomery, D. (1994): Citizen-Worker: The Experience of Workers in the United States with Democracy and the Free Market during the Nineteenth Century, Cambridge.

Notт, J. C. (1854): Types of Mankind: or, Ethnological Researches: Based Upon the Ancient Monuments, Paintings, Sculptures, and Crania of Races, and Upon Their Natural, Geographical, Philological, and Biblical History, Philadelphia.

Pessen, E. (1967): Most Uncommon Jacksonians: The Radical Leaders of the Early Labor Movement, Albany.

ReIDy, J. P. (1992): From Slavery to Agrarian Capitalism in the Cotton Plantation South: Central Georgia, 1800-1880, Chapel Hill-London.

Reinhold, M. (1984): Classica Americana. The Greek and Roman Heritage in the United States, Detroit.

Richard, C. J. (2009): The Golden Age of the Classics in America, Greece, Rome and the Antebellum United States, Cambridge (MA).

RIVERS, W. J.

(1855): "Note", Columbia Daily South Carolinian, 27 August.

(1858): The Study of Greek Literature, Columbia S.C.

(1893): Addresses and Other Occasional Pieces, Baltimore.

Roberts, J. T. (1994): Athens on trial: The Antidemocratic Tradition in Western Thought, Princeton.

Roediger, D. (2007): The Wages of Whiteness: Race and the Making of American Working Class, London.

SKIPPER, O. C. (1958): J.D.B. Debow: Magazinist of Old South, Athens (GA).

Southron, A. (1838): “Thoughts on Slavery”, Southern Literary Messenger, December 4/12, $735-745$.

Swinton Legaré, H. (1846): “The Public Economic of Athens”, [en] M. Swinton Legaré (ed.), Writings of Hugh Swinton Legaré Consisting of a Diary of Brussels, and Journal of the Rhine, London, 502-509.

Tyse, L. E. (1987): Proslavery: A History of the Defense of Slavery in America 1701-1840, Athens (GA). 
Upshur, P. (1839): “Domestic Slavery”, Southern Literary Messenger 5/10, 677-687.

WiLTSHIRE, S. F.

(1977): "Jefferson, Calhoun and the Slavery Debate", Southern Humanities Review 11, 3340.

(1987): “Aristotle in America”, Humanities 8, 8-11.

Winterer, C. (2002): The Culture of Classicism. Ancient Greece and Rome in American Intellectual Life (1780-1910), Baltimore.

Wish, H. (1949): “Aristotle, Plato and the Maxon-Dixon Line", Journal of the History of Ideas 10, 254-266. 